\title{
Approximation Properties of de la Vallée- Poussin sums in Morrey spaces
}

\author{
Ahmed Kinj*, Mohammad Ali and Suleiman Mahmoud
}

\author{
Department of Mathematics, Faculty of Science, Tishreen University, Lattakia, Syria. *Email: \\ A.Kinj@tishreen.edu.sy.
}

\begin{abstract}
In this paper, we investigate the problem of the deviation of a function $f$ from its de la Vallée-Poussin sums of Fourier series in Morrey spaces defined on the unite circle in terms of the best approximation to $f$. Moreover, approximation properties of de la Vallée-Poussin sums of Faber series in Morrey-Smirnov classes of analytic functions, defined on a simply connected domain bounded by a curve satisfying Dini's smoothness condition are obtained.
\end{abstract}

Keywords: de la Vallée-Poussin; Faber polynomials; modulus of smoothness; Morrey Smirnov classes.

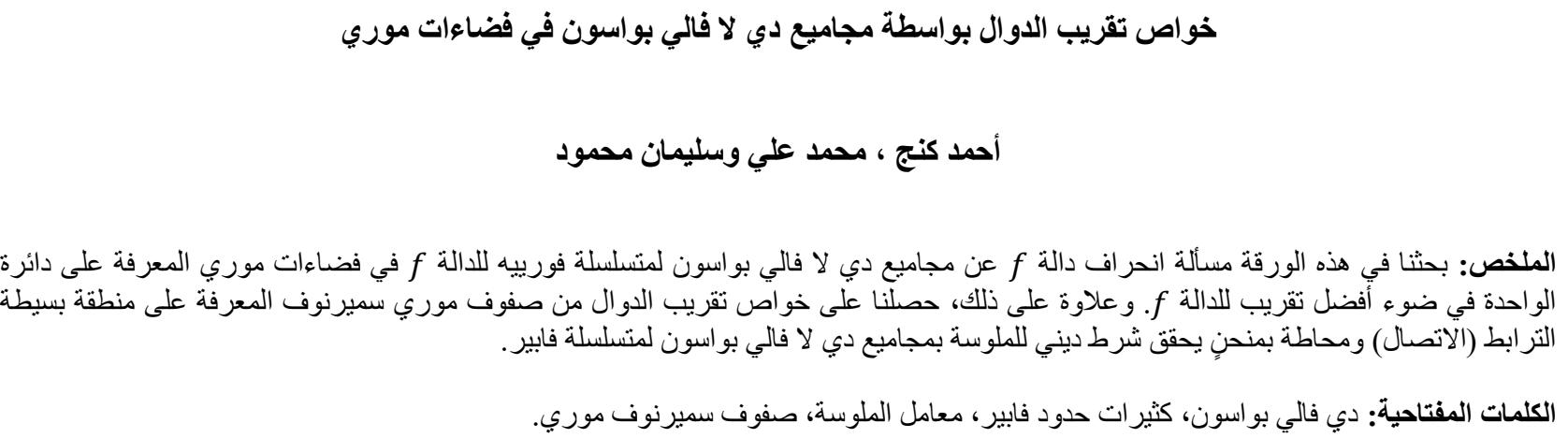

\section{Introduction}

M ain approximation problems in Lebesgue spaces have been studied by several authors $[1,2]$. The approximation of functions of Lebesgue spaces by partial sum of Faber-Laurent series was obtained by Israfilov [3]. These results are generalized to Muckenhoupt weighted Lebesgue's spaces [4]. Approximation properties of Faber series in weighted and non-weighted Orlicz spaces were dealt with by Jafarov and Israfilov [5-7].

The concept of Morrey space, introduced by C. Morrey [8] in 1938, has been studied intensively by various authors and plays an important role in many areas such as applied mathematics, the theory of differential equations, potential theory, and maximal and singular operator theory. Currently there are several investigations relating to the fundamental problems in this space [9-14]. Therefore, the investigation into the approximation of functions by means of Fourier trigonometric series in Morrey spaces is also important in these areas of research.

In the present paper, we investigate the problems of estimating the deviation of functions from their de la ValléePoussin sums in Morrey spaces. Similar results in weighted Smirnov spaces and weighted Smirnov Orlicz spaces can be found in the papers [15-17].

\section{Notation and Basic definitions}

Let $G$ be a finite simply connected domain in the complex plane $\mathbb{C}$ bounded by a rectifiable Jordan curve $\Gamma$ and $G^{-}:=\operatorname{ext} \Gamma$. Without loss of generality, we suppose that $0 \in G$. Further, let $\gamma_{0}:=\{w \in \mathbb{C}:|w|=1\}, D:=$ int $\gamma_{0}$, $D^{-}:=\operatorname{ext} \gamma_{0}$. We denote by $w=\varphi(z)$ the conformal mapping of $G^{-}$onto domain $D^{-}$normalized by the conditions

and let $\psi$ be the inverse mapping of $\varphi$.

$$
\varphi(\infty)=\infty, \quad \lim _{z \rightarrow \infty} \frac{\varphi(z)}{z}>0,
$$

We begin with the following definitions: 
Definition 2.1 [18] For $0 \leq \alpha \leq 2$ and $1 \leq p<\infty$, we denote by $L^{p, \alpha}(\Gamma)$ the Morrey space, as the set of locally integrable function $f$, with a finite norm:

$$
\|f\|_{L^{p, \alpha}(\Gamma)}:=\left\{\sup _{B} \frac{1}{|B \cap \Gamma|_{\Gamma}^{1-\frac{\alpha}{2}}} \int_{B \cap \Gamma}|f(z)|^{p}|d z|\right\}^{\frac{1}{p}}<\infty,
$$

where $B$ is an arbitrary disk centered on $\Gamma$ and $|B \cap \Gamma|_{\Gamma}$ is the linear Lebesgue measure of the set $B \cap \Gamma$.

In the case of $\Gamma=\gamma_{0}:=\{w \in \mathbb{C}:|w|=1\}$ we obtain the space $L^{p, \alpha}\left(\gamma_{0}\right)$.

Under this definition $L^{p, \alpha}(\Gamma)$ is a Banach space. If $\alpha=2$ then the class $L^{p, 2}(\Gamma)$ coincides with the class $L^{p}(\Gamma)$, and for $\alpha=0$ the class $L^{p, 0}(\Gamma)$ coincides with the class $L^{\infty}(\Gamma)$. Moreover, $L^{p, \alpha_{1}}(\Gamma) \subset L^{p, \alpha_{2}}(\Gamma)$ for $0 \leq \alpha_{1} \leq \alpha_{2}$. Thus, $L^{p, \alpha}(\Gamma) \subset L^{1}(\Gamma), \forall \alpha \in[0,2]$.

For given $f \in L_{1}\left(\gamma_{0}\right)$, let

$$
\frac{a_{0}}{2}+\sum_{k=0}^{\infty} a_{k}(f) \cos k x+b_{k}(f) \sin k x
$$

be the Fourier series of $f$, where $a_{k}(f)$ and $b_{k}(f)$ are Fourier coefficients of the function $f$. Further, let

$$
S_{n}(x, f)=\frac{a_{0}}{2}+\sum_{k=0}^{n} a_{k}(f) \cos k x+b_{k}(f) \sin k x
$$

be the $n$th partial sums of series (1).

We define the $n-t h$ de la Vallée-Poussin sums of series (1) as

$$
V_{n, m}(x, f)=\frac{1}{m+1} \sum_{k=n-m}^{n} S_{k}(x, f), 0 \leq m \leq n, \quad m, n=1,2,3, \ldots .
$$

Definition 2.2 [19] We define the $r$ modulus of smoothness of a function $f \in L^{p, \alpha}\left(\gamma_{0}\right)$ for $r=1,2,3$, ...by the relation

where

$$
\omega_{p, \alpha}^{r}(f, t):=\sup _{|h| \leq t}\left\|\Delta_{h}^{r}(f, .)\right\|_{L^{p, \alpha}\left(\gamma_{0}\right), \quad t>0,}
$$

$$
\Delta_{h}^{r}(f, x)=\sum_{k=0}^{r}\left(\begin{array}{l}
r \\
k
\end{array}\right)(-1)^{r-k} f(x+k h) .
$$

The best approximation to $L^{p, \alpha}\left(\gamma_{0}\right)$ in the class $\mathcal{T}_{n}$ of trigonometric polynomials of degree not greater than $n$ is defined by

$$
E_{n}(f)_{L^{p, \alpha}\left(\gamma_{0}\right)}:=\inf \left\{\left\|f-T_{n}\right\|_{L^{p, \alpha}\left(\gamma_{0}\right)}: T_{n} \in \mathcal{T}_{n}\right\} .
$$

Let $T^{*} \in \mathcal{T}_{n}$ be a trigonometric polynomial such that

$$
E_{n}(f)_{L^{p, \alpha}\left(\gamma_{0}\right)}:=\left\|f-T^{*}\right\|_{L^{p, \alpha}\left(\gamma_{0}\right)} .
$$

If $m, n \in \mathbb{N}$ such that $m \geq n$, then we get

$$
E_{m}(f)_{L^{p, \alpha}\left(\gamma_{0}\right)} \leq E_{n}(f)_{L^{p, \alpha}}\left(\gamma_{0}\right) .
$$

Using the boundedness of operator $f \rightarrow S_{n}(., f)$ in the Morrey spaces $L^{p, \alpha}\left(\gamma_{0}\right)$ we get 


$$
\begin{gathered}
\left\|f-S_{n}(., f)\right\|_{L^{p, \alpha}\left(\gamma_{0}\right)} \leq\left\|f-T^{*}\right\|_{L^{p, \alpha}\left(\gamma_{0}\right)}+\left\|T^{*}-S_{n}(., f)\right\|_{L^{p, \alpha}\left(\gamma_{0}\right)} \\
=E_{n}(f)_{L^{p, \alpha}\left(\gamma_{0}\right)}+\left\|S_{n}\left(., f-T^{*}\right)\right\|_{L^{p, \alpha}\left(\gamma_{0}\right)} \leq E_{n}(f)_{L^{p, \alpha}\left(\gamma_{0}\right)}+C\left\|f-T^{*}\right\|_{L^{p, \alpha}\left(\gamma_{0}\right)} \\
=(C+1) E_{n}(f)_{L^{p, \alpha}\left(\gamma_{0}\right)}=c E_{n}(f)_{L^{p, \alpha}\left(\gamma_{0}\right)},
\end{gathered}
$$

where $\mathrm{C}$ is a positive constant and $c=C+1$, i.e. there exists a constant $c$ such the following relation holds

$$
\left\|f-S_{n}(., f)\right\|_{L^{p, \alpha}\left(\gamma_{0}\right)} \leq c E_{n}(f)_{L^{p, \alpha}\left(\gamma_{0}\right)} .
$$

Definition 2.3 [9] We define the Morrey-Smirnov classes $E^{p, \alpha}(G), 0 \leq \alpha \leq 2$ and $1 \leq p<\infty$, of analytic functions in $G$ as

$$
E^{p, \alpha}(G):=\left\{f \in E^{1}(G): f \in L^{p, \alpha}(\Gamma)\right\} .
$$

If we define $\|f\|_{E^{p, \alpha}(\mathrm{G})}:=\|f\|_{L^{p, \alpha}(\Gamma)}$, then $E^{p, \alpha}(G)$ becomes a Banach space.

Definition 2.4 [20] A smooth curve $\Gamma: \sigma(\mathrm{s})$ is called Dini-smooth if it satisfies the condition

$$
\int_{0}^{\delta} \frac{\Omega\left(\sigma^{\prime}(s), s\right)}{s} d s<\infty, \quad \delta>0,
$$

where $\Omega\left(\sigma^{\prime}(s), s\right)$ modulus of continuity of function $\sigma^{\prime}(s)$. By $\mathcal{D}$ we denote the set of all Dini-smooth curves.

If $\Gamma \in \mathcal{D}$, then [21]

$$
0<c_{1} \leq\left|\psi^{\prime}(w)\right| \leq c_{2}<\infty, \quad 0<c_{3} \leq\left|\varphi^{\prime}(z)\right| \leq c_{4}<\infty
$$

for some constants, $c_{1}, c_{2}, c_{3}$ and,$c_{4}$.

Hence, if $\Gamma \in \mathcal{D}$ and using (4), then by [9]

$$
f \in L^{p, \alpha}(\Gamma) \Leftrightarrow f_{0}:=f \circ \psi \in L^{p, \alpha}\left(\gamma_{0}\right)
$$

and the function $f_{0}^{+}: D \rightarrow \mathbb{C}$ defined by

$$
f_{0}^{+}(w)=\frac{1}{2 \pi i} \int_{\gamma_{0}} \frac{f_{0}(\tau)}{\tau-w} d \tau, w \in D
$$

is analytic in $D$ and $f_{0}^{+} \in E^{p, \alpha}(D)[9]$.

If $\Gamma \in \mathcal{D}$ and $r=1,2,3, \ldots$, we define the $r$ - modulus of smoothness of $f \in L^{p, \alpha}(\Gamma)$ by the relation (see, [9])

$$
\Omega_{\Gamma, p, \alpha}^{r}(f, t):=\omega_{p, \alpha}^{r}\left(f_{0}^{+}, \mathrm{t}\right), \quad t>0 .
$$

The Faber polynomials $\Phi_{k}(t)$ of degree $k$ are defined by the relation [22]

$$
\frac{\psi^{\prime}(w)}{\psi(w)-t}=\sum_{k=0}^{\infty} \frac{\Phi_{k}(t)}{w^{k+1}}, t \in \mathrm{G}, w \in D^{-} .
$$


If $f \in E^{p, \alpha}(G)$, then by the definition 2.3, $f \in E^{1}(G)$ and hence

$$
f(z)=\frac{1}{2 \pi i} \int_{\Gamma} \frac{f(s)}{s-z} d s=\frac{1}{2 \pi i} \int_{\gamma_{0}} \frac{\psi^{\prime}(w)}{\psi(w)-z} f_{0}(w) d w, \mathrm{z} \in \mathrm{G} .
$$

From the last formula and the relation (8), for every $z \in G$ we have

where

$$
f(z) \sim \sum_{k=0}^{\infty} a_{k}(f) \Phi_{k}(z), \quad z \in G,
$$

$$
a_{k}(f):=\frac{1}{2 \pi i} \int_{\gamma_{0}} \frac{f_{0}(w)}{w^{k+1}} d w, \quad k=0,1,2, \ldots .
$$

The $n-t h$ de la Vallée-Poussin sums of the series (9) are defined as

where

$$
V_{n, m}(x, f)=\frac{1}{m+1} \sum_{k=n-m}^{n} S_{k}(x, f), 0 \leq m \leq n, \quad m, n=1,2,3, \ldots,
$$

We define the operator $T$ as follows:

$$
S_{n}(z, f)=\sum_{k=0}^{n} a_{k}(f) \Phi_{k}(z)
$$

$$
\begin{gathered}
T: E^{p, \alpha}(D) \rightarrow E^{p, \alpha}(G) \\
T(f)(z):=\frac{1}{2 \pi i} \int_{\gamma_{0}} \frac{f(w) \psi^{\prime}(w)}{\psi(w)-z} d w, z \in G .
\end{gathered}
$$

In order to prove our main results, we need the following theorems.

Theorem 2.1 [10] If $\Gamma \in \mathcal{D}$, then the operator $T$ defined by (10) is linear, bounded, one to one and onto. Moreover $T\left(f_{0}^{+}\right)=f$ for $f \in E^{p, \alpha}(G)$.

Theorem 2.2 [9] Let $g \in E^{p, \alpha}(\mathrm{D})$ with $0<\alpha \leq 2$ and $1<p<\infty$. Then for a given $r=1,2,3, \ldots$ the inequality

holds with a constant $c_{5}>0$ independent of $n$.

$$
E_{n}(g)_{L^{p, \alpha}\left(\gamma_{0}\right)} \leq c_{5} \omega_{p, \alpha}^{r}\left(g, \frac{1}{n+1}\right), n=1,2,3, . .
$$

\section{Main Results}

In this section, we present the main results.

Theorem 3.1 Let $L^{p, \alpha}\left(\gamma_{0}\right)$ be a Morrey space with $0<\alpha \leq 2$ and $1<p<\infty$, then there exists a positive constant $c_{6}$ such that for any $f \in L^{p, \alpha}\left(\gamma_{0}\right), 0 \leq m \leq n, m, n=1,2, \ldots$ the inequality

is true.

$$
\left\|f-V_{n, m}(., f)\right\|_{L^{p, \alpha}\left(\gamma_{0}\right)} \leq \frac{c_{6}}{m+1} \sum_{k=n-m}^{n} E_{k}(f)_{L^{p, \alpha}\left(\gamma_{0}\right)}
$$

Proof. Let us chose the integer $j$ such that $2^{j} \leq m+1 \leq 2^{j+1}$. Then

$$
\begin{gathered}
f(x)-V_{n, m}(x, f)=\frac{1}{m+1}\left[f(x)-S_{n-m}(x, f)\right] \\
+\frac{1}{m+1}\left\{\sum_{k=1}^{j} \sum_{i=n-m+2^{k-1}}^{n-m+2^{k}-1}\left[f(x)-S_{i}(x, f)\right]\right\}+\frac{1}{m+1}\left\{\sum_{k=n-m+2^{j}}^{n}\left[f(x)-S_{k}(x, f)\right]\right\} .
\end{gathered}
$$

And from this, we get 


$$
\begin{aligned}
\| f-V_{n, m}(., f) & \|_{L^{p, \alpha}\left(\gamma_{0}\right)} \\
& \leq \frac{1}{m+1}\left\|f-S_{n-m}(., f)\right\|_{L^{p, \alpha}\left(\gamma_{0}\right)}+\frac{1}{m+1}\left\{\sum_{k=1}^{j} \sum_{i=n-m+2^{k-1}}^{n-m+2^{k}-1}\left\|f-S_{i}(., f)\right\|_{L^{p, \alpha}\left(\gamma_{0}\right)}\right\} \\
& +\frac{1}{m+1}\left\{\sum_{k=n-m+2^{j}}^{n}\left\|f-S_{k}(., f)\right\|_{L^{p, \alpha}\left(\gamma_{0}\right)}\right\} .
\end{aligned}
$$

From the relation (3), we get

$$
\begin{aligned}
& \left\|f-V_{n, m}(., f)\right\|_{L^{p, \alpha}\left(\gamma_{0}\right)} \leq \frac{c_{7}}{m+1} E_{n-m}(f)_{L^{p, \alpha}\left(\gamma_{0}\right)}+\frac{c_{8}}{m+1}\left\{\sum_{k=1}^{j} \sum_{i=n-m+2^{k-1}}^{n-m+2^{k}-1} E_{i}(f)_{L^{p, \alpha}}\left(\gamma_{0}\right)\right\}+ \\
& \frac{c_{9}}{m+1}\left\{\sum_{k=n-m+2^{j}}^{n} E_{k}(f)_{L^{p, \alpha}\left(\gamma_{0}\right)}\right\} .
\end{aligned}
$$

From (12) and using (2), we get

$$
\begin{aligned}
& \left\|f-V_{n, m}(., f)\right\|_{L, \alpha\left(\gamma_{0}\right)} \leq \frac{c_{10}}{m+1}\left\{E_{n-m}(f)_{X, \omega}+\sum_{k=1}^{j} 2^{k-1} E_{n-m+2^{k-1}}(f)_{L}{ }^{p, \alpha}\left(\gamma_{0}\right)\right\}+c_{11} \frac{1}{m+1}(m- \\
& \left.2^{j}+1\right) E_{n-m+2^{j}}(f)_{L^{p, \alpha}\left(\gamma_{0}\right)} \text {. }
\end{aligned}
$$

On the other hand, we have

$$
\begin{aligned}
& \sum_{k=1}^{j} 2^{k-1} E_{n-m+2^{k-1}}(f)_{L^{p, \alpha}\left(\gamma_{0}\right)} \leq E_{n-m+1}(f)_{L^{p, \alpha}\left(\gamma_{0}\right)}+2 \sum_{k=2}^{j} \sum_{i=n-m+2^{k-2}}^{n-m+2^{k-1}-1} E_{i}(f)_{L^{p, \alpha}\left(\gamma_{0}\right)} \leq \\
& c_{12} \sum_{k=n-m}^{n-m+2^{j-1}} E_{k}(f)_{L^{p, \alpha}\left(\gamma_{0}\right) .}
\end{aligned}
$$

Since $2^{j} \leq m+1<2^{j+1}$, we get $2^{j}>m-2^{j}+1$. Hence

$$
\left(m-2^{j}+1\right) E_{n-m+2^{j}}(f)_{L^{p, \alpha}\left(\gamma_{0}\right)} \leq \sum_{k=n-m}^{n-m+2^{j}-1} E_{k}(f)_{L^{p, \alpha}\left(\gamma_{0}\right)} .
$$

$$
\begin{aligned}
& \text { From } \\
& \qquad \begin{array}{l}
\left\|f-V_{n, m}(., f)\right\|_{L^{p, \alpha}\left(\gamma_{0}\right)} \\
\leq \frac{c_{13}}{m+1}\left\{E_{n-m}(f)_{L^{p, \alpha}\left(\gamma_{0}\right)}+\sum_{k=n-m}^{n-m+2^{j-1}} E_{k}(f)_{L^{p, \alpha}\left(\gamma_{0}\right)}+\sum_{k=n-m}^{n-m+2^{j}-1} E_{k}(f)_{L^{p, \alpha}\left(\gamma_{0}\right)}\right\} \\
\leq \frac{c_{6}}{m+1} \sum_{\substack{k=n-m \\
(11) \text { is true. }}}^{n} E_{k}(f)_{L^{p, \alpha}\left(\gamma_{0}\right)}
\end{array}
\end{aligned}
$$

and the inequality

Corollary 3.1 Let $L^{p, \alpha}\left(\gamma_{0}\right)$ be a Morrey space with $0<\alpha \leq 2$ and $1<p<\infty$, then there exists a positive constant $c_{14}$ such that for any $f \in L^{p, \alpha}\left(\gamma_{0}\right), 0 \leq m \leq n, m, n=1,2, \ldots$ the inequality

is true.

$$
\left\|f-V_{n, m}(., f)\right\|_{L^{p, \alpha}\left(\gamma_{0}\right)} \leq \frac{c_{14}}{m+1} \sum_{k=n-m}^{n} \omega_{p, \alpha}^{r}\left(f, \frac{1}{k+1}\right)
$$

Proof. From Theorem 3.1 we have

and from Theorem 2.2 we get

$$
\left\|f-V_{n, m}(., f)\right\|_{L^{p, \alpha}\left(\gamma_{0}\right)} \leq \frac{c_{6}}{m+1} \sum_{k=n-m}^{n} E_{k}(f)_{L^{p, \alpha}\left(\gamma_{0}\right)}
$$

$$
E_{n}(f)_{L}^{p, \alpha}\left(\gamma_{0}\right) \leq c_{5} \omega_{p, \alpha}^{r}\left(f, \frac{1}{n+1}\right), n=1,2,3, \ldots .
$$


We reach

$$
\left\|f-V_{n, m}(., f)\right\|_{L^{p, \alpha}\left(\gamma_{0}\right)} \leq \frac{c_{14}}{m+1} \sum_{k=n-m}^{n} \omega_{p, \alpha}^{r}\left(f, \frac{1}{k+1}\right), n=1,2, \ldots .
$$

Theorem 3.2 Let $G$ be a simply connected domain in the complex plane, bounded by a curve $\Gamma \in \mathcal{D}$. If $f \in E^{p, \alpha}(G)$ with $0<\alpha \leq 2$ and $1<p<\infty$, then for every $0 \leq m \leq n, n, m \in \mathbb{N}$ the estimate

holds, where $c_{15}$ is a positive constant.

$$
\left\|f-V_{n}(., f)\right\|_{L}^{p, \alpha}(\Gamma) \leq c_{15} \sum_{k=n-m}^{n} \Omega_{\Gamma, p, \alpha}^{r}\left(f, \frac{1}{k+1}\right)
$$

Proof. Since $f \in E^{p, \alpha}(G)$ and $\Gamma$ is a Dini - smooth curve, then the boundary function of $f$ belongs to $L^{p, \alpha}(\Gamma)$ and from the relation (5) we get $f_{0} \in L^{p, \alpha}\left(\gamma_{0}\right)$, and the function $f_{0}^{+}$which defined by (6) belongs to $E^{p, \alpha}(D)$. Since $E^{p, \alpha}(D) \subset$ $E^{1}(D)$, we obtain $f_{0}^{+} \in E^{1}(D)$ which has the following Taylor expansion

$$
f_{0}^{+}(w)=\sum_{k=0}^{\infty} a_{k}\left(f_{0}^{+}\right) w^{k}, w \in D .
$$

Let $\left\{c_{k}\right\}$ be the Fourier coefficients of the boundary function of $f_{0}^{+}$, then by [23] we get $c_{k}=a_{k}\left(f_{0}^{+}\right)$for $k \geq 0$ and $c_{k}=0$ for $k<0$, and then by substitution in (17) we obtain

$$
f_{0}^{+}(w)=\sum_{k=0}^{\infty} c_{k} w^{k}, w \in D
$$

Note that for the function $f \in E^{p, \alpha}(G)$ the following Faber series holds

$$
f(z) \sim \sum_{k=0}^{\infty} a_{k}(f) \Phi_{k}(z), \quad z \in G
$$

where $a_{k}(f), k=0,1,2, \ldots$ are the Taylor coefficients of the function $f_{0}^{+}$, and by Theorem 2.1 we obtain

and

$$
T\left(\sum_{k=0}^{n} a_{k}\left(f_{0}^{+}\right) w^{k}\right)=\sum_{k=0}^{n} a_{k}(f) \Phi_{k}(z)
$$

$$
T\left(V_{n, m}\left(\mathrm{w}, f_{0}^{+}\right)\right)=V_{n, m}(z, f), 0 \leq m \leq n, \quad n, m=0,1,2, \ldots .
$$

Hence, using the boundedness of operator $T$ defined by (10) and the relation

(11), we reach

$$
\begin{aligned}
& \left\|f-V_{n, m}(., f)\right\|_{L^{p, \alpha}(\Gamma)}=\left\|T\left(f_{0}^{+}\right)-T\left(V_{n, m}\left(., f_{0}^{+}\right)\right)\right\|_{L^{p, \alpha}(\Gamma)} \leq c_{16}\left\|f_{0}^{+}-V_{n, m}\left(., f_{0}^{+}\right)\right\|_{L^{p, \alpha}\left(\gamma_{0}\right)} \\
& \leq \frac{c_{17}}{m+1} \sum_{k=n-m}^{n} E_{k}\left(f_{0}^{+}\right)_{L^{p, \alpha}\left(\gamma_{0}\right) .}
\end{aligned}
$$

Using the Theorem 2.2 we get

And by the relation

$$
\left\|f-V_{n, m}(., f)\right\|_{L^{p, \alpha}(\Gamma)} \leq \frac{c_{15}}{m+1} \sum_{k=n-m}^{n} \omega_{p, \alpha}^{r}\left(f_{0}^{+}, \frac{1}{k+1}\right) .
$$

(7) we reach

$$
\left\|f-V_{n, m}(., f)\right\|_{L^{p, \alpha}(\Gamma)} \leq \frac{c_{15}}{m+1} \sum_{k=n-m}^{n} \Omega_{\Gamma, p, \alpha}^{r}\left(f, \frac{1}{k+1}\right) .
$$

Consequently, we have proved the Theorem 3.2. 


\section{Conclusion}

A method was developed to estimate the deviation of functions from their de la Vallée-Poussin sums in Morrey spaces in terms of the best approximation.

\section{References}

1. Guven, A. Trignometric approximation of function in weighted Lp spaces. Sarajevo Journal of Math, 2009, 5(17), 99-108.

2. Andersson, J. On the degree of polynomial approximation in $E^{p}(D)$. Journal of Approximation Theory, 1977, 19(1), 61-68.

3. Çavu, A. and Israfilov, D. Approximation by Faber-Laurent rational functions in the mean of functions of class $\mathrm{L}_{\mathrm{p}}(\Gamma)$ with $1<\mathrm{p}<+\infty$. Approximation Theory and its Applications, 1995, 11(1), 105-118.

4. Israfilov, D. Approximation by p-Faber-Laurent rational functions. Czechoslovak Mathematical Journal, 2004, 54(129), 751-765.

5. Jafarov, S. Approximation by polynomials and rational functions in Orlicz spaces. Journal of computational analysis and applications, 2011, 13(5), 953-962.

6. Jafarov, S. On approximation in weighted Smirnov Orlicz classes. Complex Variables and Elliptic Equations, 2012, 57(5), 567-577.

7. Israfilov, D. and Akgun, R. Approximation in weighted Smirnov- Orlicz classes. Journal of Math. Kyoto University., 2006, 46(4), 755-770.

8. Morrey, C. On the solutions of quasi-linear elliptic partial differential equations. Transactions of the American Mathematical Society, 1938, 43(1), 126-166.

9. Israfilov, D. and Tozman, N. Approximation by polynomials in Morrey-Smirnov classes. East Journal on, Approximation, 2008, 14(3), 225-269.

10. Israfilov, D. and Tozman, N. Approximation in Morrey-Smirnov classes. Azerbaijan Journal of Math., 2011, 1(1), 99-113.

11. Bilalov, B. and Quliyeva, A. On basicity of exponential systems in Morrey-type spaces. International Journal of Mathematics, 2014, 25(06), 1450054 (10 pages).

12. Sadigova, S. On approximation by Shift Operators in Morrey Type Spaces. Caspian Journal of Applied Mathematics, Ecology and Economics, 2015, 3(2).

13. Bilalov, B., Gasymov, T., and Guliyeva, A. On the solvability of the Riemann boundary value problem in MorreyHardy classes. Turkish Journal of Mathematics, 2016, 40(5), 1085-1101.

14. Guliyeva, F., Abdullayeva, R., and Cetin, S. On Morrey type Spaces and Some Properties. Caspian Journal of Applied Mathematics, Ecology and Economics, 2016, 4(1), 3-16.

15. Kokilasvili, V. On approximation of analytic functions from Ep classes. Trudy Tbiliss. Razmadze Mathematical Institute, 1968, 34, 82-102.

16. Guven, A. and Israfilov, D. Approximation by mean of Fourier trignometric series in weighted Orlicz spaces. Advanced Studies in Contemporary Mathematics, 2009, 19(2), 283-295.

17. Jafarov, S. Approximation of functions by de la Vallee-Poussin sums in weighted Orlicz spaces. Arabian Journal of Mathematics, 2016, 5(3), 125-137.

18. Fucík, S., John, O., Kufner, A., and Pick, L. Function spaces: De Gruyter Series in Nonlinear Analysis and Applications 14 (2nd Edition), De Gruyter, Berlin/Boston, 2013.

19. Devore, R.A. and Lorentz, G.G. Constructive approximation: A Series of Comprehensive Studies in Mathematics, Springer, New York, 1993.

20. Pommerenke, C. Boundary Behavior of Conformal Maps: A Series of Comprehensive Studies in Mathematics, Springer, Berlin, 1992.

21. Warschawski, S.E. Uber das ranverhalten der Ableitung der Abildunggsfunktion bei Konfermer Abbildung, Math. Z. 1932, 35(German).

22. Markushevich, A. Theory of Analytic Functions, Izdatelstvo Nauka, Moscow, 1968.

23. Duren, P.L. Theory of $H^{p}$ spaces: A Series of Monographs and Textbooks, Academic Press, New York, 1970.

Received 18 October 2016

Accepted $1^{\text {st }}$ August 2017

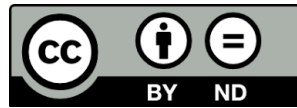

